\title{
Highly Stable 300kV Cold Field Emission Gun for 50pm Resolution Electron Microscopy
}

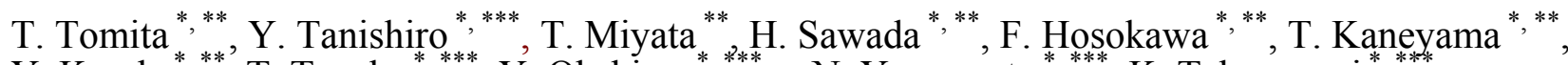 \\ Y. Kondo ${ }^{*},{ }^{* *}$, T. Tanaka ${ }^{*},{ }^{* * *}$, Y. Ohshima ${ }^{*}{ }^{* * *}$, N. Yamamoto ${ }^{*}{ }^{* * *}$, K. Takayanagi ${ }^{*}$ \\ * Japan Science and Technology Agency, CREST, 5 Sanbancho, Chiyoda-ku, Tokyo 102-0075, \\ Japan \\ *** JEOL Ltd., 3-1-2 Musashino, Akishima, Tokyo 196-8558, Japan \\ *** Department of Physics, Tokyo Institute of Technology, 2-12-1-H-51 Oh-okayama, \\ Meguro-ku, Tokyo 152-8551, Japan
}

We have been developing an ultrahigh resolution 300kV TEM / STEM, aiming at the resolution better than $50 \mathrm{pm}$ (the microscope's name is R005: Resolution $0.05 \mathrm{~nm}$ ). The spherical aberration was well corrected as reported in the previous article[1]. To obtain the target resolution, the reduction of chromatic aberration is crucial as well as the correction of the spherical aberration. Total chromatic aberration is a product of energy spread of electron source and its coefficient, which is estimated by sum of chromatic aberration coefficients for the objective lens and spherical aberration corrector in aberration corrected microscope. Thus, the low energy spread electron source must be used with an objective lens, which has a small chromatic aberration coefficient. Moreover, the high brightness and stability are required for imaging and analysis. From this point of view, we have developed a cold field emission gun, working for a long time with stable emission current at the $300 \mathrm{kV}$. The probe sizes for TFE (Thermal Field Emission), using a $\mathrm{ZrO} / \mathrm{W}$ emitter, and for CFE (Cold Field Emission) are shown in Fig. 1. For this calculation, Full width of half maximums (FWHM) of energy spreads for those are $0.8 \mathrm{eV}$ and $0.3 \mathrm{eV}$ respectively. As a result, 47pm resolution STEM image have been obtained using this CFEG [2]. This paper reports the structure and characteristics of CFEG for the R005 microscope.

For stable CF emission, the vacuum around the emitter is required to be ultrahigh enough to avoid the surface adsorption with residual gases. The adsorption induces a change of work function for emitter surface, resulting in the decrease and fluctuation of emission current. Furthermore, the ionized residual and the electron-stimulated desorption gases bomb the emitter surface, resulting in the unstable emission.[3] An emitter and an accelerator tube of the newly developed CFE gun is evacuated with the large conductance, using three sets of the NEG (Non Evaporable Getter) pumps around the emitter. Total pumping speed with these NEGs is $100 \mathrm{~L} / \mathrm{s}$ for $\mathrm{H}_{2}$, which occupies the large portion of the total residual gas at the ultrahigh vacuum level. According to the vacuum simulation, the vacuum around the emitter reaches as high as $5 \times 10^{-9} \mathrm{~Pa}$. The vacuum level, measured using an extractor gauge equipped at the bottom of accerlating tube, is shown in Fig. 2. The measured value accords with the simulated one. The vacuum has been maintained for the term longer than 100 days.

Figure 3 shows an emission pattern of W(111) after the flashing. The flashing to obtain this pattern needs several tens of seconds and performs at low temperature. Only such work requires for daily use. The stability of the emission current is less than $7 \%$ for 6 hours as shown in Fig. 4, which is enough for observation and analysis all day long. With the ultrahigh vacuum around the emitter, the decrease of emission current, which used to be found in the CFEGs, dose not take place. The measured brightness and FWHM of energy spread are around $10^{-9} \mathrm{~A} / \mathrm{cm}^{2}$ sr and $0.3 \mathrm{eV}$, respectively. The low temperature setting of the emitter promises very long lifetime of the emitter. 


\section{Reference}

[1] H. Sawada, et al.: Jpn. J. Appl. Phys., Vol. 46, No.23 (2007)

[2] H. Sawada, et al.: To be submitted elsewhere.

[3] H. Todokoro, N. Saitou and S. Yamamoto: Jpn. J. Appl. Phys., Vol. 21, 1513 (1982)

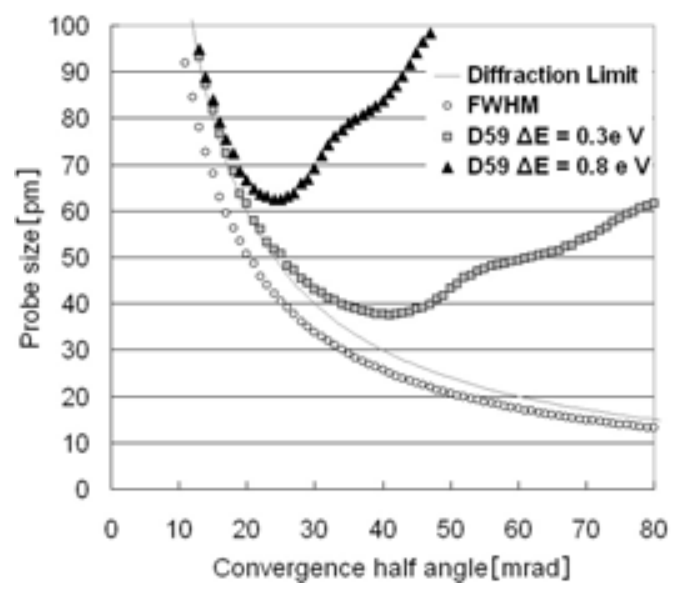

Fig. 1 The STEM probe size depending on convergent angle at $300 \mathrm{kV}$ for $\Delta \mathrm{E}=0.8 \mathrm{eV}$ and $0.3 \mathrm{eV}$

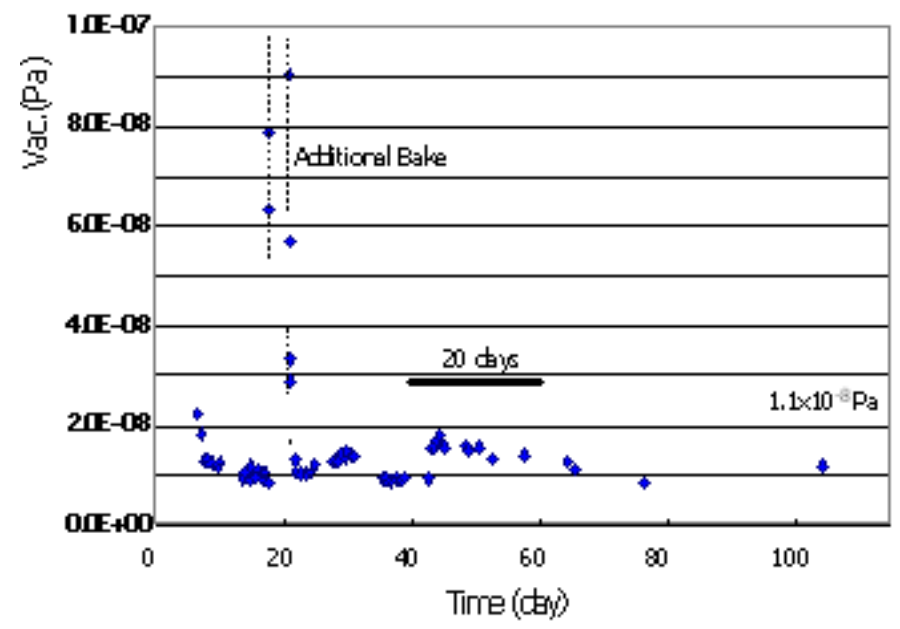

Fig.2 Pressure at the bottom of acceleration tube.

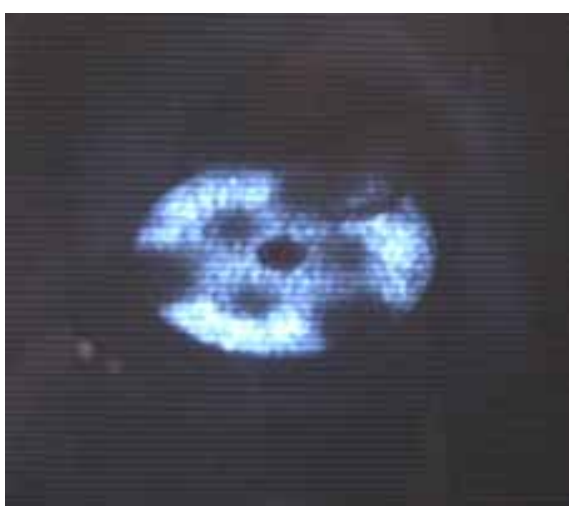

Fig.3 W(111) emission pattern after flashing.

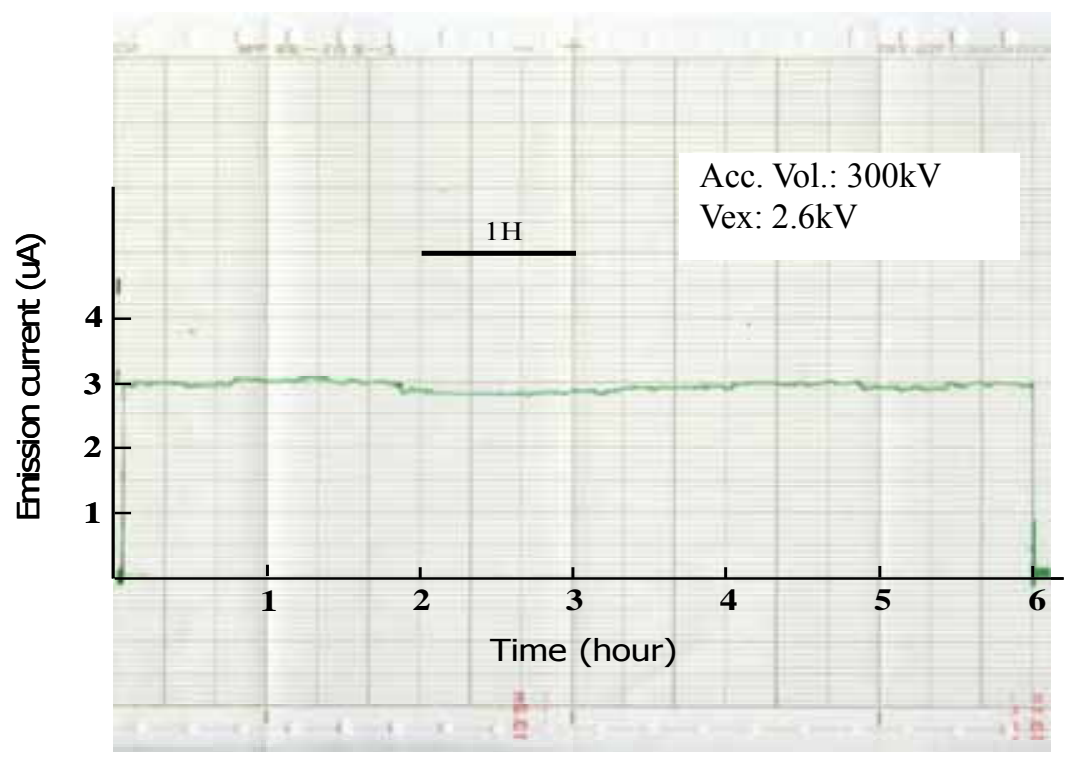

Fig.4 Emission current stability. 